Comment. Math. Helv. 76 (2001) 339-352

(C) 2001 Birkhäuser Verlag, Basel

Commentarii Mathematici Helvetici

\title{
The lower algebraic K-theory of Fuchsian groups
}

Ethan Berkove, Daniel Juan-Pineda and Kimberly Pearson

\begin{abstract}
Let $\Gamma$ be a cocompact Fuchsian group. We calculate the lower algebraic $\mathrm{K}$-theory of the integral group ring $\mathbb{Z} \Gamma$ and find an explicit formula for $\mathrm{K}_{i}(\mathbb{Z} \Gamma), i \leq 1$, in terms of the lower $\mathrm{K}$-groups of maximal finite cyclic subgroups of $\Gamma$.
\end{abstract}

Mathematics Subject Classification (2000). 19A31, 19B28, $19 \mathrm{D} 35$.

Keywords. K-theory, Fuchsian groups, Kleinian groups.

\section{Introduction}

For a ring $\mathrm{R}$ with unit, $\mathrm{K}_{i}(\mathrm{R})$ denotes the algebraic $\mathrm{K}$-groups of $\mathrm{R}$, for $i \in \mathbb{Z}$. The lower algebraic $\mathrm{K}$-theory $(i \leq 1)$ of integral group rings $\mathbb{Z} \mathrm{G}$ is reasonably understood for $\mathrm{G}$ finite, and the lower Whitehead groups $\mathrm{W} h_{i}(\mathrm{G})$ (a reduced $\mathrm{K}_{i}(\mathbb{Z G})$ ) are conjectured to be trivial for $\mathrm{G}$ torsion-free, but the groups $\mathrm{K}_{i}(\mathbb{Z} \mathrm{G})$ for $\mathrm{G}$ infinite with torsion for the most part remain unknown. Results in this latter case include work of Bass-Murthy on finitely generated abelian groups [3], Berkove-Farrell-Juan-Pearson on Bianchi groups [5], Burgisser on arithmetic groups [6], Connolly-Koźniewski, Pearson and Tsapogas on crystallographic groups ([7], [19] and [25]), Farrell-Hsiang and Farrell-Jones on virtually cyclic groups ([11] and [13]), $\mathrm{Hu}$ on some subgroups of $\mathrm{SL}_{n}\left(Q_{p}\right)$ [16] and Upadhyay on discrete subgroups of $\mathrm{SL}_{3}(\mathbb{Z})[26]$. However, few of the papers listed above contain a complete description of $\tilde{\mathrm{K}}_{0}(\mathbb{Z} \mathrm{G})$ or $\mathrm{Wh}(\mathrm{G})$ where the result is non-vanishing. In this paper we concentrate on the lower algebraic K-theory of cocompact Fuchsian groups $\Gamma$, and obtain an explicit formula for $\mathrm{W} h(\Gamma), \tilde{\mathrm{K}}_{0}(\mathbb{Z} \Gamma)$, and the negative $\mathrm{K}$-groups of $\mathbb{Z} \Gamma$ in terms of the $\mathrm{K}$-groups of the maximal finite cyclic subgroups of $\Gamma$. As $\mathrm{Wh}(\mathrm{G}), \tilde{\mathrm{K}}_{0}(\mathbb{Z} \mathrm{G})$, and $\mathrm{K}_{-1}(\mathbb{Z G})$ are usually non-trivial for finite cyclic groups $\mathrm{G}$, we consequently find the lower algebraic $\mathrm{K}$-theory of $\mathbb{Z} \Gamma$ to be non-trivial for most Fuchsian groups $\Gamma$.

A Fuchsian group is a discrete subgroup of $\mathrm{PSL}_{2}(\mathbb{R})$ or a conjugate of such a group in $\mathrm{PSL}_{2}(\mathbb{C})$. As $\mathrm{PSL}_{2}(\mathbb{R})$ can be identified with the orientation-preserving

Research partially supported by DGAPA-UNAM and CONACyT research grants 
isometries of the hyperbolic plane $\mathbb{H}^{2}$, the Fuchsian groups act in a natural way on $\mathbb{H}^{2}$. We say a Fuchsian group $\Gamma$ is cocompact if the orbit space $\mathbb{H}^{2} / \Gamma$ is compact. Farrell and Jones [12] have proved there is a significant simplification in calculating the lower algebraic K-theory of discrete cocompact subgroups of virtually connected Lie groups and their subgroups. Specifically, if $\Gamma$ is such a group, the computations of $\mathrm{K}_{i}(\mathbb{Z} \Gamma)$ for $i \leq 1$ can be reduced to a calculation based on the $\mathrm{K}$-groups of the virtually cyclic subgroups of $\Gamma$, where a group is virtually cyclic if it contains a cyclic subgroup of finite index. The cocompact Fuchsian groups provide a large class of examples to which the results of FarrellJones can be applied.

We show in this paper that a further reduction can be made: the lower algebraic $\mathrm{K}$-theory of a cocompact Fuchsian group depends on the $\mathrm{K}$-theory of its finite subgroups. Having reduced to finite subgroups, we use spectral sequences to prove our

Main Theorem. Let $\Gamma$ be a Fuchsian group such that the orbit space $\mathbb{H}^{2} / \Gamma$ is compact. Let $\left\{v_{i}\right\}$ be the vertices of $\mathbb{H}^{2} / \Gamma$ and let $\mathbb{Z} / m_{i}$ be the stabilizer of a lift $\tilde{v}_{i}$. Then

$$
\begin{aligned}
\mathrm{K}_{-1}(\mathbb{Z} \Gamma) & \cong \oplus \mathrm{K}_{-1}\left(\mathbb{Z}\left[\mathbb{Z} / m_{i}\right]\right), \\
\tilde{\mathrm{K}}_{0}(\mathbb{Z} \Gamma) & \cong \oplus \tilde{\mathrm{K}}_{0}\left(\mathbb{Z}\left[\mathbb{Z} / m_{i}\right]\right),
\end{aligned}
$$

and

$$
\mathrm{W} h(\Gamma) \cong \oplus \mathrm{Wh}\left(\mathbb{Z} / m_{i}\right) .
$$

It is well-known that given a collection of integers $\left\{g ; m_{1}, m_{2}, \ldots, m_{k}\right\}$ satisfying $m_{i} \geq 2$ and $2 g-2+\Sigma\left(1-1 / m_{i}\right)>0$, there exists a Fuchsian group $\Gamma$ and a compact fundamental polygon with $k$ vertices in $\mathbb{H}^{2}$ such that the stabilizer of the $i$ th vertex is $\mathbb{Z} / m_{i}$. Furthermore, $\mathbb{H}^{2} / \Gamma$ has genus $g$. Thus our result provides an infinite collection of examples with non-vanishing Whitehead group, non-vanishing reduced projective class group, and non-vanishing $\mathrm{K}_{-1}$. As specific examples, if any $m_{i}=5$ then $\mathrm{Wh}(\Gamma) \neq 0$, if any $m_{i}=23$ then $\tilde{\mathrm{K}}_{0}(\mathbb{Z} \Gamma) \neq 0$, and if any $m_{i}=6$ then $K_{-1}(\mathbb{Z} \Gamma) \neq 0$. Fuchsian groups $\Gamma$ with Whitehead groups of arbitrarily high rank can be produced by choosing appropriate $k$ and $m_{1}, m_{2}, \ldots, m_{k}$. Using different methods, Lück and Stamm have confirmed the main result of this paper in ([18], Theorem 1).

This paper is organized as follows: In Section 2 we recall the fundamental results of Farrell and Jones and other necessary background material. In Section 3 we find that the only possible virtually cyclic subgroups of Fuchsian groups are finite cyclic groups $\mathbb{Z} / n$, the infinite cyclic group $\mathbb{Z}$, and the infinite dihedral group $\mathbf{D}_{\infty}$. We then use this classification to prove that the lower algebraic K-theory of any cocompact Fuchsian group comes from its finite subgroups in a "spectral sequence sense." In Section 4 we perform the spectral sequence computations needed to complete the proof of the main result. 


\section{Preliminaries}

In this section we discuss the key results of Farrell-Jones and Davis-Lück needed to prove the main theorem. We begin by establishing notation and recalling some definitions.

Denote by $\mathfrak{O}(\Gamma)$ the orbit category of $\Gamma$, introduced by Bredon. Its objects are the homogeneous spaces $\Gamma / H$ regarded as left $\Gamma$-spaces, and its morphisms are $\Gamma$-maps. Denote by $\mathfrak{O}(\Gamma, \mathcal{F}) \subset \mathfrak{O}(\Gamma)$ the full subcategory consisting of objects $\Gamma / H$, where $H \in \mathcal{F}$ and $\mathcal{F}$ is a family of subgroups of $\Gamma$ closed under conjugation and inclusion. Let $\mathbb{K}: \mathfrak{O}(\Gamma) \rightarrow \Omega$-SPECTRA be the algebraic $\mathrm{K}$-theory functor of Davis-Lück [8]. A key property of this functor is that $\pi_{n}(\mathbb{K}(\Gamma / H)) \cong \mathrm{K}_{n}(\mathbb{Z} H)$. In fact, $\mathbb{K}(\Gamma / H)$ has the weak homotopy type of the classical delooping spectrum $\mathcal{K}(\mathbb{Z} H) \quad([15],[27])$. Let hocolim $\mathbb{K}$ be the homotopy colimit of the functor $\mathbb{K}$ over the category $\mathfrak{O}(\Gamma, \mathcal{F})$ as defined in [8].

Given two families $\mathcal{F} \subseteq \mathcal{F}^{\prime}$ of subgroups of $\Gamma$, the inclusion of subcategories induces a map

$$
\iota_{\mathcal{F}, \mathcal{F}^{\prime}}: \underset{\mathfrak{O}(\Gamma, \mathcal{F})}{\operatorname{hocolim}} \mathbb{K} \underset{\mathfrak{O}\left(\Gamma, \mathcal{F}^{\prime}\right)}{\operatorname{hocolim}} \mathbb{K},
$$

and corresponding maps

$$
\mathbf{A}_{\mathcal{F}, \mathcal{F}^{\prime}}: \pi_{n}(\underset{\mathcal{O}(\Gamma, \mathcal{F})}{\operatorname{hocolim}} \mathbb{K}) \rightarrow \pi_{n}\left(\underset{\mathcal{O}\left(\Gamma, \mathcal{F}^{\prime}\right)}{\operatorname{hocolim}} \mathbb{K}\right) .
$$

These are collectively known as assembly maps ([8], [12]). If we set $\mathcal{F}^{\prime}=\mathcal{F}_{\text {all }}$, the family of all subgroups of $\Gamma$, then $\mathfrak{O}\left(\Gamma, \mathcal{F}^{\prime}\right)=\mathfrak{O}(\Gamma)$ and we can identify $\pi_{n}\left(\underset{\mathfrak{O}\left(\Gamma, \mathcal{F}_{\text {all }}\right)}{\operatorname{hocolim}} \mathbb{K}\right)$ with $\mathrm{K}_{n}(\mathbb{Z} \Gamma)$ via the final object $\Gamma / \Gamma$. If in addition we set $\mathcal{F}=\mathcal{F}_{e}$, the family consisting of the trivial subgroup only, then $\mathbf{A}_{\mathcal{F}, \mathcal{F}^{\prime}}$ is the classical assembly map

$$
\mathbf{A}: H_{n}(\mathrm{~B} \Gamma ; \mathcal{K}(\mathbb{Z})) \rightarrow \mathrm{K}_{n}(\mathbb{Z} \Gamma)
$$

Assembly maps have the property that given families $\mathcal{F}_{1} \subseteq \mathcal{F}_{2} \subseteq \mathcal{F}_{3}$ of subgroups of $\Gamma$, then

$$
\mathbf{A}_{\mathcal{F}_{1}, \mathcal{F}_{3}}=\mathbf{A}_{\mathcal{F}_{2}, \mathcal{F}_{3}} \circ \mathbf{A}_{\mathcal{F}_{1}, \mathcal{F}_{2}}
$$

In general, assembly maps need not be isomorphisms, but they are key maps when trying to understand $K_{n}(\mathbb{Z} \Gamma)$ through a collection of subgroups of $\Gamma$. We focus on the family of virtually cyclic subgroups.

Definition 1. A group $\mathrm{G}$ is virtually cyclic if it contains a cyclic subgroup of finite index, i.e. if it is finite or contains a subgroup of finite index which is isomorphic to $\mathbb{Z}$.

For the rest of this paper, let $\mathcal{F}_{\mathrm{VC}}$ denote the family of virtually cyclic subgroups and $\mathcal{F}_{\text {fin }}$ the family of finite subgroups.

Let $X$ be a connected $\mathrm{CW}$-complex, and let $\mathrm{F}_{*}()$ be one of the following spectrum-valued functors: $\mathcal{P}_{*}()\left(\mathcal{P}_{*}^{\text {Diff }}()\right)$ which maps $X$ to the $\Omega$-spectrum 
of stable topological (smooth) pseudo-isotopies on $X, \mathcal{K}_{*}()$ the functor mapping $X$ to $\mathcal{K}\left(\mathbb{Z} \pi_{1} X\right)$, or $\mathcal{L}_{*}^{-\infty}()$ the $\mathrm{L}^{-\infty}$-surgery functor. In [12], Farrell and Jones introduce "The Isomorphism Conjecture," which postulates that $\mathrm{F}_{*}(X)$ can be computed in terms of spectra $\left\{\mathrm{F}_{*}\left(X_{H}\right)\right\}$, where $H$ ranges over all virtually cyclic subgroups of $\pi_{1}(X)$. In that paper Farrell and Jones also prove the conjecture for the pseudo-isotopy and smooth pseudo-isotopy functors when $\pi_{1}(X)=\Gamma$ is a subgroup of a discrete cocompact subgroup of a virtually connected Lie group. The work of Davis-Lück ([8], also see [24]) reformulates the K- and L-theoretic versions of the Isomorphism Conjecture in a manner well-suited to concrete computations. In terms of the Davis-Lück functor, the Isomorphism Conjecture in algebraic K-theory states:

Farrell-Jones Isomorphism Conjecture. The assembly map

$$
\mathbf{A}_{\mathcal{F}_{\mathrm{VC}}, \mathcal{F}_{\text {all }}}: \pi_{n}\left(\underset{\mathfrak{O}\left(\Gamma, \mathcal{F}_{\mathrm{VC}}\right)}{\operatorname{hocolim}} \mathbb{K}\right) \rightarrow \pi_{n}(\underset{\mathfrak{O}(\Gamma)}{\text { hocolim }} \mathbb{K})=\mathrm{K}_{n}(\mathbb{Z} \Gamma)
$$

is an isomorphism for all $n$.

The results of [12] yield

Farrell-Jones Theorem. Let $\Gamma$ be a subgroup of a cocompact discrete subgroup of a virtually connected Lie group. Then

$$
\mathbf{A}_{\mathcal{F}_{\mathrm{VC}}, \mathcal{F}_{\text {all }}}: \pi_{n}\left(\underset{\mathfrak{O}\left(\Gamma, \mathcal{F}_{\mathrm{VC}}\right)}{\operatorname{hocolim}} \mathbb{K}\right) \rightarrow \mathrm{K}_{n}(\mathbb{Z} \Gamma)
$$

is an isomorphism for $n \leq 1$.

The validity of the Isomorphism Conjecture for the pseudo-isotopy functor (Theorem 2.1 of [12]), together with Anderson-Hsiang's identification of the lower homotopy groups of the pseudo-isotopy spectrum and reduced lower algebraic $\mathrm{K}$ theory,

$$
\pi_{j}\left(\mathcal{P}_{*}(X)\right) \cong \mathrm{W} h_{j+2}\left(\mathbb{Z} \pi_{1}(X)\right) \text { for } j \leq-1,
$$

(Theorem 3 of [1]) implies the above theorem, essentially following the argument of 1.6.5 in [12]. Furthermore, Theorem A.8 of [12] states that if the Conjecture holds for a group $\Gamma$, then it holds for any subgroup of $\Gamma$.

Our next objective is to further reduce the problem from a computation involving the virtually cyclic subgroups of $\Gamma$ to one involving only the finite subgroups of $\Gamma$. We use the following result contained in the appendix of [12].

Theorem 2. ([12] A.10, also [26]) Let $\mathcal{F} \subseteq \mathcal{F}^{\prime}$ be two families of subgroups of $\Gamma$. For each $Q \in \mathcal{F}^{\prime}-\mathcal{F}$, define the induced family of subgroups $\mathcal{F}_{Q}$ of $Q$ as $\mathcal{F}_{Q}=\{\mathrm{G} \cap Q \mid \mathrm{G} \in \mathcal{F}\}$. The assembly map $\mathbf{A}_{\mathcal{F}, \mathcal{F}^{\prime}}$ is an isomorphism for $j<n$ and is a surjection for $j=n$ provided the assembly maps

$$
\mathbf{A}_{\mathcal{F}_{Q}, \mathcal{F}_{\text {all }(Q)}}: \pi_{j}\left(\underset{\mathfrak{O}\left(Q, \mathcal{F}_{Q}\right)}{\operatorname{hocolim}} \mathbb{K}\right) \rightarrow \mathrm{K}_{j}(\mathbb{Z} Q)
$$

are isomorphisms for all $Q \in \mathcal{F}^{\prime}-\mathcal{F}$ and all $j \leq n$. 
In order to apply Theorem 2 to the case of $\mathcal{F}=\mathcal{F}_{\text {fin }}$ and $\mathcal{F}^{\prime}=\mathcal{F}_{\text {VC }}$, our first task is to determine up to isomorphism all infinite virtually cyclic subgroups of the Fuchsian groups. Once this is established, we must check the assembly map condition given in Theorem 2 for each such subgroup. We accomplish these tasks in the next section.

\section{Reduction to Finite Subgroups}

A general description of infinite virtually cyclic groups is given in the following structure theorem due to P. Scott and C.T.C. Wall:

Theorem 3. [22] The following conditions on a finitely generated group G are equivalent:

TF1 G contains an infinite cyclic subgroup of finite index (i.e. G is an infinite virtually cyclic group).

TF2 G has a finite normal subgroup with quotient isomorphic to $\mathbb{Z}$ or $\mathrm{D}_{\infty}$.

TF3 G is isomorphic to an HNN extension of the form $\mathrm{F} \underset{\mathrm{F}}{*}$ with $\mathrm{F}$ a finite group when the quotient is $\mathbb{Z}$, and to an amalgamated product

$$
\mathrm{A} \underset{\mathrm{F}}{* \mathrm{~B}}
$$

where $\mathrm{F}$ is finite and $|\mathrm{A}: \mathrm{F}|=|\mathrm{B}: \mathrm{F}|=2$ when the quotient is $\mathbf{D}_{\infty}$.

In light of TF2, it is desirable to understand the finite subgroups of Fuchsian groups, as described by the following

Theorem 4. (see [14], 2.2.7) All finite subgroups of Fuchsian groups are cyclic, and all abelian subgroups of Fuchsian groups are cyclic.

These two results imply

Theorem 5. If $\mathrm{G}$ is an infinite virtually cyclic subgroup of a Fuchsian group $\Gamma$ then $\mathrm{G} \cong \mathbb{Z}$ or $\mathrm{G} \cong \mathbf{D}_{\infty}$.

Proof. By TF2 in Scott and Wall's theorem, G fits into an extension $1 \rightarrow \mathbb{Z} / n \rightarrow$ $\mathrm{G} \rightarrow \mathbb{Z} \rightarrow 1$ or $1 \rightarrow \mathbb{Z} / n \rightarrow \mathrm{G} \rightarrow \mathbf{D}_{\infty} \rightarrow 1$. Let $t$ be an element of infinite order in $\mathrm{G}$ and $h$ be a generator of the normal subgroup $\mathbb{Z} / n$. The element $t$ acts by conjugation on $h$ with finite order, say $k$. The subgroup $<h, t^{k}>\cong \mathbb{Z} / n \times \mathbb{Z}$ and thus cannot occur in a Fuchsian group except in the degenerate case $n=1$.

Remark 6. More involved but similar techniques can be used to classify all virtually cyclic subgroups of $\mathrm{PSL}_{2}(\mathbb{C})$, which may be of use in future computations, and we state the result here without proof. Using arguments as in [5], one can show that the possible infinite virtually cyclic subgroups of $\mathrm{PSL}_{2}(\mathbb{C})$ are the following: $\mathbb{Z}, \mathbf{D}_{\infty}, \mathbb{Z} / n \times \mathbb{Z}, \mathbb{Z} / n \rtimes \mathbb{Z}, \mathbf{D}_{n} \underset{\mathbb{Z} / n}{*} \mathbf{D}_{n}$, and $\mathbf{D}_{n} \underset{\mathbb{Z} / n}{*} \mathbb{Z} / 2 n$.

We now prove that Theorem 2 applies to any Fuchsian group $\Gamma$ with $\mathcal{F}=$ $\mathcal{F}_{\text {fin }}, \mathcal{F}^{\prime}=\mathcal{F}_{\mathrm{VC}}$, and $n=2$. Given the classification result, all that remains is to show is 
Proposition 7. The assembly map

$$
\mathbf{A}_{\mathcal{F}_{\text {fin }}, \mathcal{F}_{\text {all }}}: \pi_{j}\left(\underset{\mathfrak{O}\left(Q, \mathcal{F}_{\text {fin }}\right)}{\operatorname{hocolim}} \mathbb{K}\right) \rightarrow \mathrm{K}_{j}(\mathbb{Z} Q)
$$

is an isomorphism for all $j \leq 2$ and $Q \cong \mathbb{Z}, \mathbf{D}_{\infty}$.

Proof. As $\mathbb{Z}$ has no non-trivial finite subgroups, the assembly map above is the classical assembly map, which is well-known to be an isomorphism for $j \leq 2$.

For the case of $\mathbf{D}_{\infty} \cong \mathbb{Z} / 2 * \mathbb{Z} / 2$, the Gersten/Stallings/Waldhausen theorem for $\mathrm{K}$-groups of free products (see [2] pages 659,697 ) shows that $\mathrm{Wh}\left(\mathbf{D}_{\infty}\right)$, $\tilde{\mathrm{K}}_{0}\left(\mathbb{Z}\left[\mathbf{D}_{\infty}\right]\right)$, and the negative $\mathrm{K}$-groups of $\mathbb{Z}\left[\mathbf{D}_{\infty}\right]$ vanish. This implies the classic assembly map $\mathbf{A}_{\mathcal{F}_{e}, \mathcal{F}_{\text {all }}}$ is an isomorphism for $j \leq 1$. We claim the assembly map $\mathbf{A}_{\mathcal{F}_{e}, \mathcal{F}_{\text {fin }}}$ is also an isomorphism for $j \leq 1$. Note that $\mathbb{Z} / 2$ is the only non-trivial finite subgroup of $\mathbf{D}_{\infty}$. As $\mathbb{Z}[\mathbb{Z} / 2]$ has trivial Whitehead group, $\tilde{\mathrm{K}}_{0}$, and negative $\mathrm{K}$-groups, an application of Theorem 2 to the infinite dihedral group with $\mathcal{F}=\mathcal{F}_{e}$ and $\mathcal{F}^{\prime}=\mathcal{F}_{\text {fin }}$ yields the result. Finally, $\mathbf{A}_{\mathcal{F}_{\text {fin }}, \mathcal{F}_{\text {all }}}$ must also be an isomorphism for $j \leq 1$ since

$$
\mathbf{A}_{\mathcal{F}_{e}, \mathcal{F}_{\text {all }}}=\mathbf{A}_{\mathcal{F}_{e}, \mathcal{F}_{\text {fin }}} \circ \mathbf{A}_{\mathcal{F}_{\text {fin }}, \mathcal{F}_{\text {all }}}
$$

To complete the proof of the proposition it remains to check the assembly map condition for $Q=\mathbf{D}_{\infty}$ and $j=2$. The argument is similar to the cases $j \leq 1$ but a bit more involved. Letting $G=\mathbb{Z} / 2$, Dunwoody's calculations in [10] imply the assembly map $\mathbf{A}: H_{2}(\mathrm{G} ; \mathcal{K} \mathbb{Z}) \rightarrow \mathrm{K}_{2}(\mathbb{Z G})$ is an isomorphism. Again, since $\mathbb{Z} / 2$ is the only finite subgroup of $\mathbf{D}_{\infty}$, we apply Theorem 2 to conclude that $\mathbf{A}_{\mathcal{F}_{e}, \mathcal{F}_{f i n}}$ is an isomorphism for $Q=\mathbf{D}_{\infty}, j=2$.

Now consider the commutative diagram

$$
\begin{array}{ccc}
H_{2}(\mathbb{Z} / 2 ; \mathcal{K} \mathbb{Z}) \oplus H_{2}(\mathbb{Z} / 2 ; \mathcal{K} \mathbb{Z}) & \stackrel{\mathbf{A}}{\longrightarrow} & \mathrm{K}_{2}(\mathbb{Z}[\mathbb{Z} / 2]) \oplus \mathrm{K}_{2}(\mathbb{Z}[\mathbb{Z} / 2]) \\
i_{1} \downarrow & & i_{2} \downarrow \\
H_{2}\left(\mathbf{D}_{\infty} ; \mathcal{K} \mathbb{Z}\right) & \stackrel{\mathbf{A}}{\longrightarrow} & \mathrm{K}_{2}\left(\mathbb{Z}\left[\mathbf{D}_{\infty}\right]\right)
\end{array}
$$

where $i_{1}$ and $i_{2}$ are induced by inclusions $\mathbb{Z} / 2 \hookrightarrow \mathbf{D}_{\infty}$ on each factor, and $\mathbf{A}$ denotes assembly maps. At the group level this diagram looks like

$$
\begin{array}{ccc}
(\mathbb{Z} / 2)^{2} \oplus(\mathbb{Z} / 2)^{2} & \stackrel{\mathbf{A}}{\longrightarrow} & (\mathbb{Z} / 2)^{2} \oplus(\mathbb{Z} / 2)^{2} \\
i_{1} \downarrow & & i_{2} \downarrow \\
H_{2}\left(\mathbf{D}_{\infty} ; \mathcal{K} \mathbb{Z}\right) & \stackrel{\mathbf{A}}{\longrightarrow} & \mathrm{K}_{2}\left(\mathbb{Z}\left[\mathbf{D}_{\infty}\right]\right),
\end{array}
$$

with the top map an isomorphism. By the Gersten/Stallings/Waldhausen theorem $[28], \mathrm{K}_{2}\left(\mathbb{Z}\left[\mathbf{D}_{\infty}\right]\right) \cong(\mathbb{Z} / 2)^{3}$ and $i_{2}$ is surjective. Therefore $i_{2} \circ \mathbf{A}$ is surjective, so the bottom map is surjective, and consequently $H_{2}\left(\mathbf{D}_{\infty} ; \mathcal{K} \mathbb{Z}\right)$ is at least $(\mathbb{Z} / 2)^{3}$. The Atiyah-Hirzebruch spectral sequence shows $H_{2}\left(\mathbf{D}_{\infty} ; \mathcal{K} \mathbb{Z}\right)$ is at most $(\mathbb{Z} / 2)^{3}$. We conclude that the lower assembly map in (3) must be an isomorphism.

As in the case for $j \leq 1, \mathbf{A}_{\mathcal{F}_{e}, \mathcal{F}_{\text {fin }}}$ and $\mathbf{A}_{\mathcal{F}_{e}, \mathcal{F}_{\text {all }}}$ are isomorphisms for $Q=$ $\mathbf{D}_{\infty}, j=2$, so $\mathbf{A}_{\mathcal{F}_{\text {fin }}, \mathcal{F}_{\text {all }}}$ must be an isomorphism also. 
Applying Proposition 7 together with Theorem 2 we have

Corollary 8. Let $\Gamma$ be any cocompact Fuchsian group. Then

$$
\mathbf{A}_{\mathcal{F}_{\text {fin }}, \mathcal{F}_{\mathrm{VC}}}: \pi_{n}\left(\underset{\mathfrak{O}\left(\Gamma, \mathcal{F}_{\text {fin }}\right)}{\operatorname{hocolim}} \mathbb{K}\right) \rightarrow \pi_{n}\left(\underset{\mathfrak{O}\left(\Gamma, \mathcal{F}_{\mathrm{VC}}\right)}{\text { hocolim }} \mathbb{K}\right)
$$

is an isomorphism for all $n \leq 1$.

Furthermore, combining this corollary with the isomorphism from the FarrellJones Theorem we have the desired

Corollary 9. Let $\Gamma$ be any cocompact Fuchsian group. Then

$$
\mathbf{A}_{\mathcal{F}_{\text {fin }}, \mathcal{F}_{\text {all }}}: \pi_{n}\left(\underset{\mathfrak{O}\left(\Gamma, \mathcal{F}_{\text {fin }}\right)}{\text { hocolim }} \mathbb{K}\right) \rightarrow \mathrm{K}_{n}(\mathbb{Z} \Gamma)
$$

is an isomorphism for all $n \leq 1$.

\section{Spectral Sequence Computations}

We quickly review some results concerning Fuchsian groups that we use in this section. We state these results without proof; the interested reader should consult [4] or a similar text for details. A Fuchsian group $\Gamma$ is a discrete subgroup of $\mathrm{PSL}_{2}(\mathbb{R})$, and thus can be considered as a group of orientation-preserving isometries of the hyperbolic disc $\mathbb{H}^{2}$. The elements of finite order have geometric significance, as each maximal finite subgroup forms the stabilizer of a vertex in $\mathbb{H}^{2}$, where $\mathbb{H}^{2}$ has a simplicial decomposition determined by a fundamental polygon. Each cocompact Fuchsian group is classified by its signature, $\left\{g ; m_{1}, m_{2}, \ldots, m_{k}\right\}$, which enumerates the $k$ conjugacy classes of maximal finite subgroups $\mathbb{Z} / m_{i}$ and contains the genus $g$ of the orbit space $\mathbb{H}^{2} / \Gamma$.

Furthermore, given any collection of integers $\left\{g ; m_{1}, m_{2}, \ldots, m_{k}\right\}$ with $m_{i} \geq 2$ and $2 g-2+\Sigma\left(1-1 / m_{i}\right)>0$, there is a Fuchsian group and compact polygon in $\mathbb{H}^{2}$ such that $\mathbb{Z} / m_{i}$ are the vertex stabilizers and the quotient space has genus $g$. One can thus find cocompact Fuchsian groups which have, up to conjugacy, an arbitrary finite number of torsion elements of any order. Since the K-theory of Fuchsian groups comes from its finite subgroups, this implies that the lower algebraic K-theory of Fuchsian groups can be very complicated indeed.

We use this geometric information to compute the initial term of a spectral sequence converging to $\pi_{n}\left(\underset{\mathfrak{O}\left(\Gamma, \mathcal{F}_{\text {fin }}\right)}{\operatorname{hocolim}} \mathbb{K}\right) \cong \mathrm{K}_{n}(\mathbb{Z} \Gamma)$ and obtain our main result, stated here.

Main Theorem. Let $\Gamma$ be a Fuchsian group such that the orbit space $\mathbb{H}^{2} / \Gamma$ is compact. Let $\left\{v_{i}\right\}$ be the vertices of $\mathbb{H}^{2} / \Gamma$ and let $\mathbb{Z} / m_{i}$ be the stabilizer of a lift $\tilde{v}_{i}$. Then

$$
\begin{gathered}
\mathrm{K}_{-1}(\mathbb{Z} \Gamma) \cong \oplus \mathrm{K}_{-1}\left(\mathbb{Z}\left[\mathbb{Z} / m_{i}\right]\right), \\
\tilde{\mathrm{K}}_{0}(\mathbb{Z} \Gamma) \cong \oplus \tilde{\mathrm{K}}_{0}\left(\mathbb{Z}\left[\mathbb{Z} / m_{i}\right]\right),
\end{gathered}
$$


and

$$
\mathrm{W} h(\Gamma) \cong \oplus \mathrm{Wh}\left(\mathbb{Z} / m_{i}\right) .
$$

Additionally our proof shows $\mathrm{K}_{n}(\mathbb{Z} \Gamma)$ vanishes for all $n \leq-2$, but this is already known from ([13], 2.3).

To compute $\pi_{n}(\underset{\mathcal{O} \text { ocolim }}{\mathbb{K}})$ for all cocompact Fuchsian groups $\Gamma$ and $n \leq 1$, we use the $p$-chain spectral sequence developed by Davis and Lück in [9]. As this work is yet to appear we outline its setup. The $p$-chain spectral sequence converges to the homology of a space over a category with coefficients in a spectrum, using the homology of a space over a group as input. Technically, if $\mathrm{C}$ is a small category, $X$ a contravariant $\mathrm{C}$-space (i.e. a functor from $\mathrm{C}$ to $\{$ spaces $\}$ ) and $\mathbb{E}$ a covariant $\mathrm{C}$-spectrum, then the spectral sequence converges to $H_{n}^{\mathrm{C}}(X ; \mathbb{E})$ as defined in [8]. Our key application is in the case of the trivial $\mathrm{C}$-space $X=p t$, where the homology groups $H_{n}^{\mathrm{C}}(X ; \mathbb{E})$ are just the homotopy groups $\pi_{n}($ hocolim $\mathbb{E})$. To describe the $\mathrm{E}^{1}$-term we establish some notation.

Let $\mathrm{C}$ be a small free EI-category, i.e. a small category such that every endomorphism $x \rightarrow x$ is an isomorphism and Aut $(y)$ acts freely on $\operatorname{Mor}(x, y)$ (that is, $f \circ g=g$ implies $f=\mathrm{I} d$ ). In such a category two objects $x$ and $y$ are isomorphic if there are morphisms $x \rightarrow y$ and $y \rightarrow x$. Define a partial ordering on isomorphism classes $\bar{x} \leq \bar{y}$ if there is a morphism $x \rightarrow y$, and $\bar{x}<\bar{y}$ if there is a morphism $x \rightarrow y$ but no morphism $y \rightarrow x$. Note that the restricted orbit category $\mathfrak{O}\left(\Gamma, \mathcal{F}_{\text {fin }}\right)$ is a free EI-category, though in general $\mathfrak{O}(\Gamma)$ is not. Furthermore, $\Gamma / Q$ and $\Gamma / \mathrm{K}$ are isomorphic in $\mathfrak{O}\left(\Gamma, \mathcal{F}_{\text {fin }}\right)$ if and only if $Q$ and $\mathrm{K}$ are conjugate, and $\overline{\Gamma / Q} \leq \overline{\Gamma / \mathrm{K}}$ if and only if $Q$ is subconjugate to $\mathrm{K}$.

Definition 10. The sequence

$$
c=\left\{\bar{x}_{0}<\bar{x}_{1}<\ldots<\bar{x}_{p}\right\}
$$

is called a $p$-chain. For any $p$-chain $c$ with $p \geq 2$, define

$$
\begin{gathered}
\mathrm{S}(c)=\operatorname{Mor}\left(x_{p-1}, x_{p}\right) \underset{\operatorname{Aut}\left(x_{p-1}\right)}{\times} \operatorname{Mor}\left(x_{p-2}, x_{p-1}\right) \underset{\operatorname{Aut}\left(x_{p-2}\right)}{\times} \cdots \underset{\operatorname{Aut}\left(x_{1}\right)}{\times} \operatorname{Mor}\left(x_{0}, x_{1}\right), \\
\mathrm{S}(c)=\operatorname{Mor}\left(x_{0}, x_{1}\right) \text { if } c \text { is a 1-chain, and } \mathrm{S}(c)=\operatorname{Aut}\left(x_{0}\right) \text { if } c \text { is a 0-chain. }
\end{gathered}
$$

In the orbit category, a $p$-chain can be thought of as a chain of subgroups $Q_{0}<Q_{1}<\ldots<Q_{p}$ of $\Gamma$, with each $Q_{i}$ strictly subconjugate to $Q_{i+1}$. However, to emphasize that we are working with homogeneous spaces, we continue to write objects in the category as quotients $\Gamma / Q$. For simplicity we suppress notation and write $\Gamma / Q$ for $\overline{\Gamma / Q}$.

The $p$-chain spectral sequence is defined in [9] in generality for any small category $\mathrm{C}$, but there are significant simplifications when $\mathrm{C}$ is a free EI-category and we state that case here. We also specialize to the special case of a trivial Cspace, when the sequence converges to the homotopy colimit. 
Theorem 11. [9] Let $\mathrm{C}$ be a small free EI-category and $\mathbb{E}$ a covariant Cspectrum. Then there exists a spectral sequence

$$
\mathrm{E}_{p, q}^{1}=\bigoplus_{p-\text { chains } c} H_{q}^{\operatorname{Aut}\left(x_{0}\right)}\left(p t \underset{\operatorname{Aut}\left(x_{p}\right)}{\times} \mathrm{S}(c) ; \mathbb{E}\left(x_{0}\right)\right) \Rightarrow \pi_{p+q}(\underset{\mathrm{C}}{\operatorname{hocolim}} \mathbb{E}) .
$$

This is not an Atiyah-Hirzebruch type spectral sequence (as that in 8.7 of [21] or 4.7 of [8]), but generalizes the spectral sequence of Lück ([17], 17.18 and 17.28), essentially replacing the $\mathrm{RC}$-modules $\mathrm{M}$ and $N$ by a $\mathrm{C}$-space $X$ and $\mathrm{C}$-spectrum $\mathbb{E}$ in the fashion of ([8], Section 2). In this analogy, $\operatorname{Tor}_{n}^{\mathrm{RC}}(\mathrm{M}, N)$ becomes $H_{n}^{\mathrm{C}}(X ; \mathbb{E})$. Another spectral sequence of this type is described in [23].

For our computations we apply Theorem 11 using $\mathrm{C}=\mathfrak{O}\left(\Gamma, \mathcal{F}_{\text {fin }}\right)$ with coefficients in the $\mathrm{C}$-spectrum $\mathbb{K}$. Then

$$
\mathrm{E}_{p, q}^{1}=\bigoplus_{p-\text { chains } c} H_{q}^{\operatorname{Aut}\left(\Gamma / Q_{0}\right)}\left(p t \underset{\operatorname{Aut}\left(\Gamma / Q_{p}\right)}{\times} \mathrm{S}(c) ; \mathbb{K}\left(\Gamma / Q_{0}\right)\right) \Rightarrow \pi_{p+q}\left(\underset{\mathfrak{O}\left(\Gamma, \mathcal{F}_{f i n}\right)}{\text { hocolim }} \mathbb{K}\right) .
$$

When $\Gamma$ is a cocompact Fuchsian group the $\mathrm{E}^{1}$-term can be described as follows.

Proposition 12. Let $c=\left\{\Gamma / Q_{0}<\Gamma / Q_{1}<\cdots<\Gamma / Q_{p}\right\}$ be a $p$-chain in $\mathfrak{O}\left(\Gamma, \mathcal{F}_{\text {fin }}\right)$, and fix a fundamental polygon in $\mathbb{H}^{2}$ for $\Gamma$. Then if $Q_{p} \neq e$ there exists a unique vertex $v$ in $\mathbb{H}^{2} / \Gamma$ such that $\Gamma / Q_{p} \leq \Gamma / \mathrm{V}$ where $\mathrm{V}$ is the stabilizer of a lift of $v$. The summand of the $\mathrm{E}_{p, q}^{1}$-term in the $p$-chain spectral sequence corresponding to $c$ is

$$
\begin{gathered}
H_{q}(\mathrm{~B} \Gamma ; \mathcal{K}(\mathbb{Z})), \text { if } Q_{0}=e, p=0 \\
H_{q}\left(\mathrm{~B}\left(\mathrm{~V} / Q_{0}\right) ; \mathcal{K}\left(\mathbb{Z} Q_{0}\right)\right), \text { otherwise. }
\end{gathered}
$$

Here $\mathcal{K}(\mathrm{R})$ denotes Gersten's algebraic $\mathrm{K}$-theory spectrum [15]. Note in the special case of a 0-chain $c=\Gamma / \mathrm{V}$, the last line simplifies to $\mathrm{K}_{q}(\mathbb{Z V})$.

Proof. Let $H$ be any non-trivial finite subgroup of $\Gamma$ and $\mathrm{V}$ be a maximal finite subgroup containing $H$. As $\mathrm{V}$ is the stabilizer of a lift of some vertex in $\mathbb{H}^{2} / \Gamma$ and the stabilizers of edges are trivial, each $H$ must be contained in a unique V . Furthermore (see Corollary 10.3.3 in [4]), $N(H) \cong \mathrm{V}$, where $N(H)$ denotes the normalizer in $\Gamma$ of $H$. It is then straightforward to check that in the orbit category, $\operatorname{Aut}(\Gamma / H)=\mathrm{V} / H$ and if $\Gamma / H<\Gamma / H^{\prime}$ then $\operatorname{Mor}\left(\Gamma / H, \Gamma / H^{\prime}\right)=$ $\mathrm{V} / H^{\prime}$. Consequently, most of the terms of $\mathrm{S}(c)$ collapse. There are slightly different results depending on whether or not $Q_{0}=e$.

If $Q_{0} \neq e$, then $\mathrm{S}(c)=\mathrm{V} / Q_{p}$ and the component of $\mathrm{E}_{p, q}^{1}$ corresponding to 
$c$ is

$$
\begin{gathered}
\mathrm{E}_{p, q}^{1}=H_{q}^{\operatorname{Aut}\left(\Gamma / Q_{0}\right)}\left(p t \underset{\operatorname{Aut}\left(\Gamma / Q_{p}\right)}{\times} \mathrm{S}(c) ; \mathbb{K}\left(\Gamma / Q_{0}\right)\right) \\
=H_{q}^{\mathrm{V} / Q_{0}}\left(p t \underset{\mathrm{V} / Q_{p}}{\times} \mathrm{V} / Q_{p} ; \mathbb{K}\left(\Gamma / Q_{0}\right)\right) \\
=H_{q}^{\mathrm{V} / Q_{0}}\left(p t ; \mathbb{K}\left(\Gamma / Q_{0}\right)\right) \\
\cong H_{q}\left(\mathrm{~B}\left(\mathrm{~V} / Q_{0}\right) ; \mathbb{K}\left(Q_{0} / Q_{0}\right)\right) \\
=H_{q}\left(\mathrm{~B}\left(\mathrm{~V} / Q_{0}\right) ; \mathcal{K}\left(\mathbb{Z} Q_{0}\right)\right) .
\end{gathered}
$$

The isomorphism from equivariant to regular homology above is induced by the natural $\mathrm{V} / Q_{0}$-equivariant map $\mathbb{K}\left(\Gamma / Q_{0}\right) \rightarrow \mathbb{K}\left(Q_{0} / Q_{0}\right)$, with trivial action on the target (see [19], 3.7 and [8], 5.5).

If $Q_{0}=e$, then $\operatorname{Aut}\left(\Gamma / Q_{0}\right)=\Gamma$ and $\operatorname{Mor}\left(\Gamma / Q_{0}, \Gamma / Q_{1}\right)=\Gamma / Q_{1} ;$ thus $\mathrm{S}(c)$ simplifies to $\mathrm{V} / Q_{p} \underset{\mathrm{V} / Q_{1}}{\times} \Gamma / Q_{1}$. For $p>0$, the corresponding summand in $\mathrm{E}_{p, q}^{1}$ is

$$
\begin{gathered}
\mathrm{E}_{p, q}^{1}=H_{q}^{\operatorname{Aut}\left(\Gamma / Q_{0}\right)}\left(p t \underset{\operatorname{Aut}\left(\Gamma / Q_{p}\right)}{\times} \mathrm{S}(c) ; \mathbb{K}\left(\Gamma / Q_{0}\right)\right) \\
=H_{q}^{\Gamma}\left(p t_{\mathrm{V} / Q_{p}}^{\times}\left(\mathrm{V} / Q_{p} \underset{\mathrm{V} / Q_{1}}{\times} \Gamma / Q_{1}\right) ; \mathbb{K}(\Gamma / e)\right) \\
=H_{q}^{\Gamma}(\Gamma / \mathrm{V} ; \mathbb{K}(\Gamma / e)) \\
\cong H_{q}(\mathrm{BV} ; \mathbb{K}(e / e)) \\
\cong H_{q}(\mathrm{BV} ; \mathcal{K}(\mathbb{Z})) .
\end{gathered}
$$

In the degenerate case $p=0$,

$$
H_{q}^{\Gamma}(p t ; \mathbb{K}(\Gamma / e)) \cong H_{q}(\mathrm{~B} \Gamma ; \mathbb{K}(e / e)) \cong H_{q}(\mathrm{~B} \Gamma ; \mathcal{K}(\mathbb{Z})) .
$$

In the next two propositions, we show that for Fuchsian groups the $p$-chain spectral sequence collapses at $\mathrm{E}^{2}$, and we compute the $\mathrm{E}^{2}=\mathrm{E}^{\infty}$-term.

Proposition 13. Let $\Gamma$ be any cocompact Fuchsian group. Then $\mathrm{E}_{p, q}^{2}=0$ if $p \geq 2$ in the $p$-chain spectral sequence and thus the spectral sequence collapses at $\mathrm{E}^{2}$.

Proof. We start by giving some background about the differential $d_{p, q}^{1}: \mathrm{E}_{p, q}^{1} \rightarrow$ $\mathrm{E}_{p-1, q}^{1}$ in the $p$-chain spectral sequence as described in [9] and ([17], 17.27 and $17.28)$. Let $d$ be a $p$-chain and let $c$ be a $(p-1)$-chain that arises from $d$ by omitting some non-initial object; let $y_{p}$ and $x_{p-1}$ denote the final objects of $d$ and $c$. There is a natural map from $\mathrm{S}(d)$ to $\mathrm{S}(c)$ through composition of the appropriate terms in $\mathrm{S}(d)$, or by dropping the appropriate morphism if $c$ is obtained by omitting the final object of $d$ (defined up to Aut $\left(x_{p-1}\right)$ ). This yields a map

$$
p t \underset{\operatorname{Aut}\left(y_{p}\right)}{\times} \mathrm{S}(d) \rightarrow p t \underset{\operatorname{Aut}\left(x_{p-1}\right)}{\times} \mathrm{S}(c) .
$$


The induced homomorphism on homology, together with a plus or minus sign arising from the standard degeneracy operator at the level of chains, is the $d^{1}$ differential on the summands of $\mathrm{E}^{1}$ corresponding to $d$ and $c$. If $c$ is obtained by omitting the initial object of $d$, then the differential on the corresponding summands is more complex as it involves a change of coefficients, but we do not need this case to prove the Proposition. If $c$ is not a chain obtained by omitting some object of $d$, then the differential restricted to those summands is zero.

There is a useful relationship between chains in $\mathfrak{D}\left(\Gamma, \mathcal{F}_{\text {fin }}\right)$ when $\Gamma$ is a cocompact Fuchsian group. Let $p \geq 1, c$ be a $p$-chain such that $Q_{p}$ is not a maximal finite subgroup, and $c+\mathrm{V}$ be the $(p+1)$-chain obtained by adding to $c$ the maximal finite subgroup $\mathrm{V}$ containing $Q_{p}$, so that

$$
\begin{gathered}
c=\left\{\Gamma / Q_{0}<\Gamma / Q_{1}<\cdots<\Gamma / Q_{p}\right\}, \\
c+\mathrm{V}=\left\{\Gamma / Q_{0}<\Gamma / Q_{1}<\cdots<\Gamma / Q_{p}<\Gamma / Q_{p+1}=\Gamma / \mathrm{V}\right\} .
\end{gathered}
$$

Recall from Proposition 12 that if $Q_{p} \neq e$, then there is a unique maximal finite subgroup $\mathrm{V}$ containing $Q_{p}$. This uniqueness guarantees that for $p \geq 1$ there is a one-to-one correspondence between such chains $c$ and $c+\mathrm{V}$. First suppose $Q_{0} \neq e$. By Proposition 12, the $\mathrm{E}^{1}$ summands corresponding to $c+\mathrm{V}$ and $c$ are both equal to $H_{q}^{\mathrm{V} / Q_{0}}\left(p t ; \mathbb{K}\left(\Gamma / Q_{0}\right)\right)$. The $d^{1}$ differential is induced by the trivial map $p t \rightarrow p t$, and is plus or minus the identity when restricted to these summands. Similarly, if $Q_{0}=e$, then $d_{1}$ restricted to the summands corresponding to $c+\mathrm{V}$ and $c$ is also (plus or minus) the identity.

Write $\mathrm{E}_{p, q}^{1}=\mathrm{A}_{p, q} \oplus \mathrm{B}_{p, q}$, where $\mathrm{A}$ (resp. B) consists of the summands arising from $p$-chains such that $Q_{p}$ is a maximal finite subgroup (resp. $Q_{p}$ is not maximal). Take an element $x \in \mathrm{E}_{p, q}^{1}, p>0$, and express it as $x=a+b$, with $a \in \mathrm{A}_{p, q}, b \in \mathrm{B}_{p, q}$. As $b$ arises from some sum of chains $\gamma$, create a new sum of $(p+1)$-chains $\gamma^{\prime}$ by adding the appropriate maximal $\mathrm{V}$ to the end of each chain in $\gamma$. Since $d^{1}$ restricted to $\mathrm{E}^{1}$ summands of the type $c$ and $c+\mathrm{V}$ described above is plus or minus the identity, we can choose a class $a^{\prime} \in \mathrm{A}_{p+1, q}$ arising from $\gamma^{\prime}$ such that $d^{1}\left(a^{\prime}\right)=b+a^{\prime \prime}$, with $a^{\prime \prime} \in \mathrm{A}_{p, q}$. This follows from the action of the degeneracy operator; when it is applied to a $(p+1)$-chain ending with a maximal $\mathrm{V}$, all but one of the resulting $p$-chains will end in $\mathrm{V}$ as well. Then

$$
a+b=a+\left(d^{1}\left(a^{\prime}\right)-a^{\prime \prime}\right)=\left(a-a^{\prime \prime}\right)+d^{1}\left(a^{\prime}\right) .
$$

Thus any element $x$ of $\mathrm{E}_{p, q}^{1}$ can be expressed as a sum $x=a+d^{1}\left(a^{\prime}\right)$ with $a \in \mathrm{A}_{p, q}, a^{\prime} \in \mathrm{A}_{p+1, q}$.

We complete the proof by showing that $d^{1}$ is injective when restricted to $\mathrm{A}_{p, q}$ for $p \geq 2$. A class of the form $(a, 0) \in \mathrm{A}_{p, q} \oplus \mathrm{B}_{p, q}=\mathrm{E}_{p, q}^{1}$ has a non-zero component arising from a chain $c+\mathrm{V}$ if and only if $d^{1}(a, 0)$ has a non-zero component in the summand corresponding to $c$. There can be no cancellation of such terms since if $c^{\prime}+\mathrm{V}^{\prime} \neq c+\mathrm{V}$, then $d^{1}\left(c^{\prime}+\mathrm{V}^{\prime}\right)$ is zero on the $c$ summand. Again this is a consequence of each non-trivial finite subgroup of $\Gamma$ being contained 
in a unique maximal finite subgroup. Thus no non-trivial class of the form $(a, 0)$ is in the kernel of $d^{1}$.

Therefore the intersection of $\mathrm{A}_{p, q}$ and $d^{1}\left(\mathrm{~A}_{p+1, q}\right)$ is trivial and we can write $\mathrm{E}_{p, q}^{1}=\mathrm{A}_{p, q} \oplus d^{1}\left(\mathrm{~A}_{p+1, q}\right)$ for $p \geq 2$. As an immediate consequence, $\mathrm{E}_{p, q}^{2}$ vanishes for $p \geq 2$ and the spectral sequence collapses at $\mathrm{E}^{2}$.

Proposition 14. Let $\Gamma$ be any cocompact Fuchsian group. Then in the p-chain spectral sequence converging to $\pi_{p+q}\left(\underset{\mathfrak{O}\left(\Gamma, \mathcal{F}_{\text {fin }}\right)}{\text { hocolim }} \mathbb{K}\right) \cong \mathrm{K}_{p+q}(\mathbb{Z} \Gamma)$ we have

$$
\begin{aligned}
\mathrm{E}_{1, q}^{2} & =0, q \leq 1, \\
0 \rightarrow \Gamma^{a b} \oplus \mathbb{Z} / 2 \rightarrow \mathrm{E}_{0,1}^{2} & \rightarrow \oplus_{i} \mathrm{Wh}\left(\mathbb{Z} / m_{i}\right) \rightarrow 0, \\
0 \rightarrow \mathbb{Z} \rightarrow \quad \mathrm{E}_{0,0}^{2} & \rightarrow \oplus_{i} \tilde{\mathrm{K}}_{0}\left(\mathbb{Z}\left[\mathbb{Z} / m_{i}\right]\right) \rightarrow 0, \\
\mathrm{E}_{0,-1}^{2} & =\oplus_{i} \mathrm{~K}_{-1}\left(\mathbb{Z}\left[\mathbb{Z} / m_{i}\right]\right), \\
\text { and } \quad \mathrm{E}_{0, q}^{2} & =0 \text { for } q \leq-2 .
\end{aligned}
$$

Proof. We need only determine $d^{1}: \mathrm{E}_{1, q}^{1} \rightarrow \mathrm{E}_{0, q}^{1}$ to complete the proof. As in the proof of Proposition 13, we write $\mathrm{E}_{1, q}^{1,}=\mathrm{A}_{1, q}+d^{1}\left(\mathrm{~A}_{2, q}\right)$, and show that $d_{1, q}^{1}$ restricted to $\mathrm{A}_{1, q}$ is injective provided $q \leq 1$ (the range in which we are interested). The case $\{\Gamma / Q<\Gamma / \mathrm{V}\} \rightarrow \Gamma / Q$ when $Q \neq e$ is covered by Proposition 13 , and terms corresponding to these chains do not survive to $\mathrm{E}^{2}$. Next consider differentials arising from chains of the type $\{\Gamma / e<\Gamma / \mathrm{V}\} \rightarrow \Gamma / \mathrm{V}$, where we have $d_{1, q}^{1}: H_{q}(\mathrm{BV} ; \mathcal{K}(\mathbb{Z})) \rightarrow \mathrm{K}_{q}(\mathrm{~V})$. Following the general description in [9] and ([17], $17.27)$, the differential in this particular case is shown in $([19], 3.10)$ to be the classic assembly map, which is injective for $q \leq 1$. Therefore $d^{1}$ restricted to $\mathrm{A}_{1, q}$ is injective for $q \leq 1$ and so $\mathrm{E}_{1, q}^{2}$ vanishes for $q \leq 1$.

From the description of $\mathrm{E}_{0, q}^{1}$ in Proposition 12 and the above analysis of $d^{1}$, we have

$$
0 \rightarrow \oplus_{i} H_{q}\left(\mathrm{BV}_{i} ; \mathcal{K}(\mathbb{Z})\right) \rightarrow H_{q}(\mathrm{~B} \Gamma ; \mathcal{K}(\mathbb{Z})) \oplus\left(\oplus_{i} \mathrm{~K}_{q}\left(\mathbb{Z} \mathrm{V}_{i}\right)\right) \rightarrow \mathrm{E}_{0, q}^{2} \rightarrow 0,
$$

where the maps from $H_{q}\left(\mathrm{BV}_{i} ; \mathcal{K}(\mathbb{Z})\right)$ are assembly maps and inclusions on homology, and $i$ runs over conjugacy classes of maximal finite subgroups $\mathrm{V}_{i}$. As $\mathrm{V}_{i} \cong \mathbb{Z} / m_{i}$ is abelian, $\mathrm{K}_{1}\left(\mathbb{Z V}_{i}\right) \cong \mathrm{V}_{i} \oplus \mathbb{Z} / 2 \oplus \mathrm{Wh}\left(\mathrm{V}_{i}\right)$ and the image of the assembly map $H_{1}\left(\mathrm{BV}_{i} ; \mathcal{K}(\mathbb{Z})\right) \rightarrow \mathrm{K}_{1}\left(\mathbb{Z V}_{i}\right)$ splits for each $i$. As $H_{1}(\mathrm{BG} ; \mathcal{K}(\mathbb{Z})) \cong$ $\mathrm{G}^{a b} \oplus \mathbb{Z} / 2$ in general, we get a short exact sequence

$$
0 \rightarrow \Gamma^{a b} \oplus \mathbb{Z} / 2 \rightarrow \mathrm{E}_{0,1}^{2} \rightarrow \oplus_{i} \mathrm{Wh}\left(\mathrm{V}_{i}\right) \rightarrow 0 .
$$

When $q=0$ we obtain

$$
0 \rightarrow \mathbb{Z} \rightarrow \mathrm{E}_{0,0}^{2} \rightarrow \oplus_{i} \tilde{\mathrm{K}}_{0}\left(\mathbb{Z}\left[\mathbb{Z} / m_{i}\right]\right) \rightarrow 0
$$

the $\mathrm{E}^{2}$-term described in the proposition.

Finally, the standard reductions from $\mathrm{K}_{0}$ to $\tilde{\mathrm{K}}_{0}$ and from $\mathrm{K}_{1}$ to Whitehead groups immediately yield our main result. 
Remark 15. The calculations can also be performed using Quinn's spectral sequence $([21], 8.7)$. One obtains the same results for $\tilde{\mathrm{K}}_{0}$ and the negative $\mathrm{K}$ groups, but a non-zero $d^{2}$ differential prevents the determination of the Whitehead group. For Fuchsian groups, that spectral sequence collapses at $\mathrm{E}^{3}$.

\section{Acknowledgements}

We kindly thank all those who have assisted with the realization of this work, especially Jim Davis and Wolfgang Lück for their advice and making their results accessible to us, Tom Farrell for his support and encouragement, and the referee for valuable suggestions which improved the manuscript.

\section{References}

[1] D. R. Anderson, W.-C. Hsiang, The functors $\mathrm{K}_{-i}$ and pseudo-isotopies of polyhedra Ann. of Math. 105 (1999), 210-223.

[2] H. Bass, Algebraic K-Theory, W. A. Benjamin, Inc., New York, 1968.

[3] H. Bass and M. P. Murthy, Grothendieck groups and Picard groups of abelian group rings, Ann. of Math. 86 (1967), 16-73.

[4] A. F. Beardon, The Geometry of Discrete Groups, Springer-Verlag, Graduate Texts in Mathematics 91, 1983.

[5] E. Berkove, F. T. Farrell, D. Juan-Pineda, K. Pearson, The Farrell-Jones isomorphism conjecture for finite co-volume hyperbolic actions and the algebraic K -theory of Bianchi groups, Trans. Amer. Math. Soc. 352 (2000), 5689-5702.

[6] B. Bürgisser, On the projective class group of arithmetic groups, Math. Z. 184 (1983), 339-357.

[7] F. Connolly, T. Koźniewski, Rigidity and crystallographic groups, I, Invent. Math. 99 (1990), 25-48

[8] J. F. Davis, W. Lück, Spaces over a category and assembly maps in isomorphism conjectures in K- and L-theory, K-Theory 15 (1998), 201-252.

[9] J. F. Davis, W. Lück, Computations of K-and L-groups of group rings based on isomorphism conjectures, to appear.

[10] M. J. Dunwoody, $\mathrm{K}_{2}(\mathbb{Z} \Pi)$ for $\Pi$ a group of order two or three, J. London Math. Soc. (2), 11 (1975), 481-490.

[11] F. T. Farrell, W.-C. Hsiang, A formula for $\mathrm{K}_{1} \mathrm{R}_{\alpha}[\mathrm{T}]$, Proc. Symp. Pure Math. vol. 17, Applications of Categorical Algebra, American Mathematical Society, Providence, 1970.

[12] F. T. Farrell, L. Jones, Isomorphism conjectures in algebraic K-theory, J. Amer. Math. Soc. 6 no 2 (1993), 249-298.

[13] F. T. Farrell, L. Jones, Lower algebraic K-theory of virtually infinite cyclic groups, $K$ Theory 9 (1995), 13-30.

[14] B. Fine, Algebraic theory of the Bianchi Groups, Marcel Dekker, Monographs and textbooks in pure and applied mathematics; 129, 1989.

[15] S. Gersten, On the spectrum of algebraic K-theory, Bull. Amer. Math. Soc. 78 (1972), 216-219.

[16] B. Hu, Whitehead groups of finite polyhedra with non-positive curvature, J. Differential Geom. 38 no. 3 (1993), 501-517.

[17] W. Lück, Transformation Groups and Algebraic K-Theory, Lecture Notes in Mathematics, volume 1408, Springer-Verlag, 1989. 
[18] W. Lück, R. Stamm, Computations of K - and L -theory of cocompact planar groups, K - Theory 21 (2000), 249-292.

[19] K. Pearson, Algebraic K -theory of two-dimensional crystallographic groups, K -Theory 14 (1998), 265-280.

[20] E. Pedersen, C. Weibel, A non-connective delooping of algebraic K-theory, Topology Lecture Notes in Math., vol. 1126, Springer-Verlag, Berlin-Heidelberg-New York, 166$181,1985$.

[21] F. Quinn, Ends of maps II, Invent. Math. 68 (1982), 353-424.

[22] P. Scott, C.T.C. Wall, Topological methods in group theory, Homological Group Theory, Proceedings held in Durham in September 1977, Ed. C. T. C. Wall, London Mathematical Society Lecture Note Series, volume 36, 1979.

[23] J. Slomińska, Equivariant Bredon cohomology of classifying spaces of families of subgroups, Bull. Acad. Polon. Sciences, Vol XXVIII, 503-508, 1980.

[24] R. Talbert, An isomorphism between Bredon and Quinn homology, Forum Math. 11 no. 5 (1999), 591-616.

[25] G. Tsapogas, On the K -theory of crystallographic groups, Trans. Amer. Math. Soc. 347 8 (1995).

[26] S. Upadhyay, Controlled algebraic $\mathrm{K}$-theory of integral group ring of $\mathrm{SL}(3, \mathbb{Z}), \mathrm{K}$ Theory 10 no. 4 (1996), 413-418.

[27] J. Wagoner, Delooping classifying spaces in algebraic K-theory, Topology 11 (1972), 349-370.

[28] F. Waldhausen, Algebraic K -theory of generalized free products, Parts I and II, Ann. of Math. 108 (1978), 135-204 and 205-256.

Ethan Berkove

Lafayette College

Department of Mathematics

Easton, PA 18042

USA

e-mail: berkovee@lafayette.edu

Daniel Juan-Pineda

Instituto de Matemáticas

UNAM

Campus Morelia

Apartado Postal

61-3 XANGARI

Morelia Michoacán

MEXICO 58089

e-mail: daniel@matmor.unam.mx

Kimberly Pearson

Valparaiso University

Dept. of Math. \& CS.

Valparaiso, IN 46383

USA

e-mail: kpearson@valpo.edu

(Received: November 10, 1998; revised version: August 11, 2000) 